\title{
FRAÇÃO IDEAL DA CARGA HORÁRIA COM AULAS DE CAMPO, LABORATÓRIO E SALA NO ENSINO DE SOLOS: VISÃO DO ALUNO
}

\section{Allocation of time for lessons between field, laboratory and classroom in soils' teaching: students' views}

Arno Rieder ${ }^{1}$

\begin{abstract}
Resumo: O tempo ideal (CH) preconizado por estudantes, para aulas de campo (AC), sala (AS) e de laboratório (AL) no ensino sobre solos no nível Fundamental (1G), Médio (2G) e Superior (3G), foi investigado em três escolas de Cáceres e Cuiabá, MT, Brasil. Foram avaliados os efeitos do nível escolar dos opinantes sobre a $\mathrm{CH}$ indicada. Houve efeito diferencial da escolaridade dos opinantes sobre a $\mathrm{CH}$ preconizada para as aulas AC e AS destinada para 1G. Para as aulas AC a $\mathrm{CH}$, foi maior que para AS e AL nas três escolaridades. Houve tendência de os opinantes de níveis mais adiantados sugerirem a diminuição da $\mathrm{CH}$ para $\mathrm{AC}$ aplicadas aos níveis escolares anteriores. Conclui-se que as aulas sobre solos, em qualquer nível escolar, segundo os estudantes, devem ser desenvolvidas em AC, AS e AL, utilizando uma fração diferenciada de $\mathrm{CH}$ entre ambientes de aula, com predominância para AC. Os resultados subsidiam o ajustamento do ensino sobre solos.
\end{abstract}

Palavras-chave: Solos. Ensino. Ambiente de aula. Carga horária.

\begin{abstract}
The ideal time (CH) recommended by students for field classes (AC), classroom (AS) and laboratory (AL) in teaching about soils at the fundamental (1G), second (2G) and higher (3G) levels was investigated in three schools of Cáceres and Cuiabá-MT, Brazil. We evaluated the effects of educational level of respondents on the $\mathrm{CH}$ indicated. There was a differential effect of schooling of the respondents on the $\mathrm{CH}$ recommended to $\mathrm{AC}$ and $\mathrm{AS}$ designed to 1G. For the $\mathrm{CH}$ class $\mathrm{AC}$ was greater than for AS and AL in all three education levels. Responders to more advanced school levels tend to suggest the reduction of $\mathrm{CH}$ for $\mathrm{AC}$ shown earlier school levels. We conclude that the classes on soils, at any grade level, according to students, should be developed in AC, AS and AL, using a different $\mathrm{CH}$ between classroom environments, predominantly $\mathrm{AC}$. The results provide for the adjustment of time for teaching about soils.
\end{abstract}

Keywords: Soils. Teaching. Classroom environment. Class-time. Students' opinion.

\footnotetext{
${ }^{1}$ Universidade do Estado de Mato Grosso (UNEMAT), Departamento de Matemática, Av. São João, s/n, Cavalhada, CEP 78200-000, Caceres, MT, Brasil. E-mail: arno@unemat.br
} 
Rieder, A.

\section{Introdução}

Há necessidade de rever a educação formal periodicamente em seus fundamentos, estrutura e procedimentos (DORIN, 1978; FERREIRA, 1988; MARCOS, 1988; MELLO, 1979; PENTEADO, 1980; REICHARDT, 1988; RUELLAN, 1988; TAVARES, 1960; TRANI, 1988).

No Brasil, o tema "solos" é abordado de algum modo no Ensino Fundamental (1G), Médio (2G) e Superior (3G), e pode ser ministrado em três ambientes: sala, laboratório e campo. A fração da carga-horária total por ambiente varia devido a vários fatores: projeto didático-pedagógico, nível escolar, eficácia no processo, resposta e pleitos, entre outros.

O estudo do solo é essencial para a humanidade (REICHARDT, 1988; RUELLAN, 1988). Falhas no ensino comprometem esta interação (RUELLAN, 1988) e, consequentemente, também o desenvolvimento sustentado.

O ensino de solos apresenta numerosos desafios, conforme mencionam trabalhos de Beck e Klamt (1988), Costa e Dias (1988), Ruellan (1988), Lima (2005), Frasson e Werlang (2010). Problemas na educação formal no Brasil são gerais e, no ensino de solos, são indicadas algumas soluções já por décadas (ARAÚJO; CONDE; LUZIO, 2004; BRANDÃO, 1981; CECCON; OLIVEIRA; OLIVEIRA, 1982; DORIN, 1978; GOLGHER, 2010; TAVARES, 1960). Para galgar êxito em educação ambiental, é básico o ensino de solos. As pessoas, segundo a educação recebida, têm vigorado seu poder transformador da realidade e da sociedade (MUGGLER; PINTO SOBRINHO; MACHADO, 2006).

O ensino de solos no nível Fundamental e Médio tem muitos adeptos (DINIZ; BATISTA; SANTOS, 2005). Reichardt (1988) e Trani (1988) defendem o estudo do solo num nível mais razoável de detalhe, já no Ensino Fundamental e Médio $\left(1^{\circ}\right.$ e $2^{\circ}$ graus - Lei de Diretrizes e Bases para o Ensino, LDBEN 5692/71), pois apenas uma pequena e privilegiada parcela da população brasileira alcança o Ensino Superior. No Brasil, em 1995, foram oferecidas 610.355 vagas para ingresso no Ensino Superior para 2.653 .853 inscritos em $1^{\mathrm{a}}$ opção no vestibular (INEP, 2011).

Apesar da proposição de algumas noções sobre solos no Ensino Fundamental, por meio da oferta de conteúdo de alguns livros-textos, como, por exemplo, de Silva e Fontinha (19-) e Silva Junior, Sasson e Sanches (1995), uma série de ações precisou ser implementada no Brasil, para a educação produzir melhores resultados. Iniciativas nos últimos anos têm sido tomadas por algumas equipes - como exemplo, a das Universidades Federais do Paraná e Viçosa, conforme Muggler, Pinto Sobrinho e Machado (2006), assim como o kit de material didático criado por Falconi (2004) e o material didático sobre solos ao Ensino Médio e técnico proposto por Catanozi (2004) - que visam melhorar o ensino de solos. Um retrato atual mostra que conteúdos sobre solos já estão mais contemplados em considerável número de livros didáticos destinados mais à parte final do Ensino Fundamental, embora ainda haja importantes desafios pela frente, sobretudo para as séries mais iniciais.

O desejado desenvolvimento sustentado brasileiro requer que o solo seja bem tratado, precisando ser mais bem conhecido por cada cidadão. Pouco resolve instituir avanços textuais no Código Florestal do Brasil sem melhorar ações educativas que levem em conta a constituição complexa do ambiente, que tem como uma das principais matrizes o solo. As futuras gerações só se mostrarão eficazes na gestão ambiental se forem apropriadamente edu- 
cadas e, para isto, o ensino sobre solos é primordial, desde a Educação Básica. O solo bem tratado é a matriz de sustentação ao atendimento da demanda crescente da humanidade, sobretudo por alimentos.

O conhecimento propiciado pela educação precisa ter um significado para as pessoas envolvidas. No caso de solos, isto é facilmente suscitável, em especial por ser um fator fundamental na relação sustentável do homem no ambiente. Muggler, Pinto Sobrinho e Machado (2006) enfatizam que o conhecimento significativo é fundamental para a sua construção.

A eficácia do ensino de solos é afetada por vários fatores. A distribuição da carga horária com os eventos didático-pedagógicos é um destes. O ensino deve ser estimulante e não tedioso para o aluno e, a carga horária inadequadamente distribuída pode provocar frustração, e baixa eficácia na formação do cidadão.

Embora o ensino formal sobre solos possa utilizar três ambientes para desenvolver o processo de ensino-aprendizagem (campo; sala de aula; laboratório), predominantemente, é utilizado o ambiente sala de aula e, às vezes, exclusivamente. Os pais de alunos do Ensino Fundamental podem constatar isto ao acompanharem o estudo de seus filhos. E, às vezes, ao se proporem adequações aos professores nas escolas, diagnosticam-se entraves, como: despreparo do professor, inadequações técnico-administrativas e de gestão. A própria experiência de vida do autor deste trabalho, seja como aluno, pai ou docente, testemunha a predominância de aulas em sala no ensino de solos.

A experiência anterior do indivíduo, aproveitada no processo de ensino-aprendizagem escolar, torna mais significativo o resultado educativo. A convivência ou o contato prévio com a realidade facilitam as reflexões multifacetadas e o entendimento dos alunos sobre o tema, em especial, se orientados por um professor preparado. A aula de campo, praticada após as aulas teóricas, em geral dadas em sala, possui poder de consolidar mais as mensagens educativas. Entre outros autores, Compiani e Dal Ré Carneiro (1993) aprofundam discussões para se refletir sobre os papéis didáticos de ambientes de aula.

Para Alves, Elóy e Pereira (2009), a aula de campo em educação ambiental complementa o conhecimento iniciado em sala, instigando a percepção dos alunos, e a consciência ambiental nestes. Quando desenvolvidas em ambientes naturais, podem ser mais eficazes por envolverem e motivarem os alunos nas atividades educativas, por superarem a fragmentação dos conteúdos, e aprimorarem a relação homem-natureza (SENICIATO; CAVASSAN, 2008); o ensinoaprendizagem aperfeiçoa-se, também, pelo impacto estético, instigando, emocionalmente, os que interagem com o fato real em sua integridade (FONSECA; CALDEIRA, 2008). Os aspectos estéticos são importantes, em especial, para alunos do Ensino Fundamental (SENICIATO; SILVA; CAVASSAN, 2006). A inserção favorece o entendimento do objeto de estudo em seu meio, propiciando riqueza de detalhes à construção do conhecimento, suscitando reflexões amplas e inter-relação, o que diminui o problema da fragmentação do conhecimento.

As aulas de campo são positivas aos alunos e professores, estimulando-os às atividades interdisciplinares, em equipe de docentes, quebrando rotinas e gerando inovações (PRIEVE; LISOSVKI, 2010). Os alunos relacionam-se afetivamente com o ambiente vivenciado, comprometendo-se com questões ambientais; passam a conhecer aspectos e elementos do meio e reconhecem a importância da preservação ambiental (CAPUCHO, 2009). As aulas de campo são fundamentais na educação integral dos alunos em todos os níveis. Contribuem na promoção da cidadania e da consciência ambiental. 
Tratando de formação de docentes, Righetto (2007) sugere que as ações no ensino devam ser criativas, inovadoras e com a teoria e prática adequadamente articuladas.

Constatações em Lima (2010) indicam que as aulas de laboratório no ensino de solos, são aplicadas, predominantemente, para propiciar o aprofundamento analítico do tema. Nessas utilizam-se recursos instrumentais e metodológicos que potencializam e clareiam as análises dos alunos; servem para treinar na operação metodológica e instrumental no laboratório.

Estudos de Caon (2005) revelam que aulas de laboratório e de experimentação são essenciais em Ciências e Biologia, embora não sejam habituais no dia a dia escolar, por algumas razões apontadas por docentes, como: falta de gosto; carga horária elevada; conteúdo extenso; limitações na qualidade executiva de experimentos; grande número de turmas e numerosas, o que desestimula professores a utilizarem laboratórios para suas aulas. Professores consideram importantes estas aulas, mas precisam ser bem preparadas e motivar o aluno a envolver-se, e, por fim, para construir um novo conhecimento.

Beck e Klamt (1988), ao reformularem o conteúdo no ensino de solos na graduação (Agronomia) da Universidade Federal do Rio Grande do Sul (UFRGS), introduziram uma fração de dois terços da carga horária total com estágios no meio rural. Os primeiros resultados indicaram dificuldades e resistências às inovações, limitando mais e melhores práticas.

Pessoa (2007) notou queixa de alunos de Ensino Médio referente à distribuição dos tipos de aulas em Geografia; pediam mais interação no mundo real: mais aulas de campo e práticas.

O tempo usado para aulas de campo, em sala e laboratório estimula, distintamente, a aprendizagem dos alunos. Entre educandários ocorrem variações na carga-horária destinada para uma mesma disciplina, o que também foi constatado por Midão e Ruiz-Moreno (2010).

As bases usadas para distribuição de tempo de aula por ambiente, raramente, levam em conta a opinião dos alunos. Embora as sugestões destes possam não estar tão bem fundamentadas quanto às dos educadores, não podem ser desprezadas. Conforme Rezende e Pires (2009), conhecer a visão do aluno revela seu pensamento, naquela fase da vida, sobre a educação que recebe. Suas percepções servem de subsídio à reflexão dos educadores.

Para compreender o estudante e encaminhá-lo no processo de ensino-aprendizagem, segundo Dorin (1978), há de se considerar: a hierarquia motivacional, conflitos, frustrações, mecanismos de ajustamento, as diferenças entre grupos etários, os efeitos da docência e da estrutura escolar, que impactam o indivíduo e o grupo.

A definição do tempo ideal para aulas em cada ambiente de ensino, no caso de solos, pode ser mais acertada se forem consideradas, criticamente, as opiniões de estudantes egressos. Pois podem revelar falhas nas estratégias de ensino a que forem submetidos e, oportunizar correções.

Estas opiniões podem ser dinâmicas no tempo e no espaço geográfico. Para comparar as manifestações de alunos com relação ao ensino de solos em diferentes épocas, é necessário produzir estudos periódicos neste sentido. 
O presente trabalho revela opiniões de estudantes do Ensino Fundamental, Médio e Superior, com relação ao tempo ideal que deveria ser aplicado ao estudo de solos, nas aulas em ambiente de campo (AC), de sala de aula (AS) e de laboratório (AL).

O estudo retrata opiniões de alunos de vários anos passados (1995), porém considera-se oportuna a publicação destes resultados em qualquer momento, para servir de referência a outros trabalhos e para estudos periódicos nesta temática. Os resultados contribuem para: estimular reflexões; sugerir aprofundamento e novos focos investigativos na temática; subsidiar ajustes didático-pedagógicos, revisões de projetos, programas e políticas educacionais para aprimoramento.

\section{Material e método}

Os dados do estudo foram coletados em Cuiabá e Cáceres, Mato Grosso (MT), Brasil.

$\mathrm{Na}$ época da coleta de campo de dados, os níveis de ensino, conforme a Lei de Diretrizes e Bases da Educação Brasileira (BRASIL, 1971), eram categorizados em: Primeiro (1G), Segundo (2G) e Terceiro Graus (3G), correspondendo à denominação da atual LDBEN (BRASIL, 1996), de Ensino Fundamental, Médio, e Superior, respectivamente.

O trabalho foi desenvolvido com base na aplicação orientada de formulário (Apêndice I) junto a estudantes do ensino: Fundamental (1G) [da $8^{a}$ série: 25 alunos] do $1^{\circ}$ Grau do Instituto Santa Maria (Escola Particular); Médio (2G) [da $3^{\text {a }}$ série: 48 alunos] do curso de Técnico em Agropecuária - $2^{\circ}$ Grau da Escola Agrotécnica Federal, ambas em Cáceres (MT), e Superior (3G) [do $8^{\circ}, 9^{\circ}$ e $10^{\circ}$ semestres: 33 alunos] do curso de Engenharia Agronômica da Universidade Federal de Mato Grosso, em Cuiabá (MT).

O período de coleta das informações se deu no mês de outubro de 1995.

Os estudantes amostrados ocasionalmente (presença em sala) foram alunos egressos de disciplinas que envolviam ensino de solos, nos três níveis escolares (1G, $2 \mathrm{G}$ e $3 \mathrm{G}$ ).

O formulário (Apêndice I) constou de perguntas sobre a identidade e a opinião do informante a respeito da distribuição ideal da carga horária $(\mathrm{CH})$ para três ambientes de aula (campo-AC, sala-AS e laboratório-AL), em três níveis de ensino sobre solos. Opinaram, também, sobre a $\mathrm{CH}$ que deve ser usada nos níveis escolares já concluídos pelos mesmos.

Estudantes do Ensino Superior ( $3 G$ ) opinaram sobre a CH ideal para seu nível (3G) e para os anteriores já cursados (1G, 2G); os do Ensino Médio opinaram para o seu nível (2G) e para o anterior $(1 \mathrm{G})$; os do Ensino Fundamental opinaram apenas para seu próprio nível (1G).

Este estudo constitui-se de três fatores, cada um em três níveis: Ambiente de aulas (AC, AS, AL); Estudantes opinantes (1G, 2G, 3G); Ensino-alvo das opiniões (1G, 2G, 3G). A variável de mensuração é a fração (\%) de carga horária sugerida para aulas.

A análise estatística dos dados foi efetuada em programas computadorizados (SAEG; SPSS), determinando: distribuições de dados; medidas de tendência central e de dispersão, e, também, análise de variância e testes de média $(\alpha<0,05)$. 
Rieder, A.

\section{Resultados e discussão}

\section{Distribuição da carga horária no ensino de solos (Tabela 1)}

\section{Por ambiente de aula}

Sala, laboratório e campo são ambientes complementares para desenvolver aulas e alcançar os resultados desejados na educação através do processo de ensino-aprendizagem.

$\mathrm{O}$ ambiente em que ocorre o processo ensino-aprendizagem influencia o resultado educativo. Isto se evidencia quando o tema de aula é um ente inserido e articulado na natureza, como é o caso do estudo de solos e de sua utilização. Aulas como de solos em ambientes naturais são mais eficazes na aprendizagem e, inclusive, permitem interligar conteúdos de várias áreas do conhecimento, o que também mencionam Seniciato e Cavassan (2008).

O ensinar-aprender pode ser muito mais eficaz ao se utilizarem imagens e, mais ainda, se as aulas forem dadas com o próprio objeto de estudo, na presença de seu ambiente de ocorrência. Os alunos logo geram a imagem própria do objeto de estudo e do cenário envolvido e as associam às demais informações recebidas de conteúdo. Esta criação se fixa mais firmemente na mente, como um conhecimento novo construído, polido, com sentido estético, bem armazenado e disponível para se articular a qualquer momento na reflexão e uso humano.

Um estudo de Seniciato, Silva e Cavassan (2006) também constatou o poder estético na aprendizagem nas aulas de ciências desenvolvidas em ambientes naturais.

\section{AC - Aulas no campo}

As aulas desenvolvidas no próprio ambiente do objeto de estudo motivam e tornam mais interessante o estudo. Quando adequadamente conduzidas, possibilitam aos aprendizes captarem os dados e informações do meio real para a construção do conhecimento novo.

Aulas de campo em ambientes naturais são mais eficazes que aulas teóricas em sala, o que é corroborado por vários autores (FONSECA; CALDEIRA, 2008; PRIEVE; LISOSVKI, 2010; SENICIATO; CAVASSAN, 2008). Essas permitem destacar a paisagem, os componentes dos cenários, a estética ambiental, o que sensibiliza e atrai mais a atenção dos alunos, sobretudo os em idade de Ensino Fundamental. Seniciato, Silva e Cavassan (2006) também enfatizam o valor estético das aulas de campo.

As aulas de campo são mais desejadas. Estimulam o aluno à aprendizagem e facilitam a função do professor (CAPUCHO, 2009; PESSOA, 2007; SENICIATO; SILVA; CAVASSAN, 2006).

\section{AC.1. Entre as opiniões para um mesmo nível escolar-alvo}

Para o Ensino Fundamental (alvo: 1G), houve divergência entre os três níveis de opinantes $(1 \mathrm{G}, 2 \mathrm{G}, 3 \mathrm{G})$. Os opinantes $1 \mathrm{G}$ sugeriram uma fração da $\mathrm{CH}$ maior para aulas AC $(51,5 \%)$ em relação aos opinantes $2 \mathrm{G}(37,4 \%)$ e $3 \mathrm{G}(31,8 \%)$ [Tab.1.1]. Enquanto para o Ensino Médio (alvo: $2 \mathrm{G}$ ) os dois níveis de opinantes ( $2 \mathrm{G}$ e $3 \mathrm{G}$ ) indicaram uma fração de $\mathrm{CH}$ similar, embora os valores sinalizem provável tendência de decréscimo com o aumento da

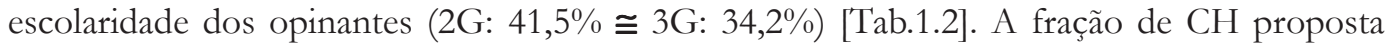
pelos opinantes 3G para seu próprio nível (alvo: 3G - Agronomia) foi de 41,5\% [Tab.1.3]. 
Fração ideal da carga horária ...

Tabela 1*. Opinião de estudantes sobre a fração ideal de carga horária para aulas de campo, em sala e em laboratório na docência do tema solos em três níveis de ensino (1G=Fundamental; 2G=Médio; 3G=Superior), Cuiabá e Cáceres-MT, 1995

\begin{tabular}{|c|c|c|c|c|}
\hline & \multirow[b]{3}{*}{ Opinantes $\Rightarrow$ alvos } & \multicolumn{3}{|c|}{ Carga horária - $\mathrm{CH}(\%)$ ideal sugerida por ambiente e nível de ensino } \\
\hline & & \multicolumn{3}{|c|}{ Ambiente de ensino } \\
\hline & & AC (Campo) & AS (Sala) & AL (Laboratório) \\
\hline \multicolumn{5}{|c|}{ Tab. 1.1. Para aulas de solos em $(\Rightarrow) 1 \mathrm{G}$ (Ensino Fundamental) } \\
\hline $1 \mathrm{G}$ & $\Rightarrow 1 \mathrm{G}$ & $51,52 \mathrm{Aa}$ & $23,08 \mathrm{~B} \mathrm{~b}$ & $25,40 \mathrm{Ab}$ \\
\hline $2 \mathrm{G}$ & $\Rightarrow 1 \mathrm{G}$ & $37,46 \mathrm{~B}$ a & $34,09 \mathrm{AB}$ a b & $28,43 \mathrm{Ab}$ \\
\hline $3 G$ & $\Rightarrow 1 \mathrm{G}$ & $31,81 \mathrm{~B} \mathrm{~b}$ & $43,94 \mathrm{Aa}$ & $24,24 \mathrm{Ab}$ \\
\hline \multicolumn{5}{|c|}{ Tab. 1.2. Para aulas de solos em $(\Rightarrow)$ 2G (Ensino Médio) } \\
\hline $2 \mathrm{G}$ & $\Rightarrow 2 \mathrm{G}$ & $41,57 \mathrm{Aa}$ & $26,73 \mathrm{~B} \mathrm{~b}$ & $31,69 \mathrm{Ab}$ \\
\hline $3 G$ & $\Rightarrow 2 \mathrm{G}$ & $34,29 \mathrm{~A}$ a b & $37,94 \mathrm{~A} \mathrm{a}$ & $27,74 \mathrm{Ab}$ \\
\hline \multicolumn{5}{|c|}{ Tab. 1.3. Para aulas de solos em $(\Rightarrow) 3 G$ (Ensino Superior) } \\
\hline $3 G$ & $\Rightarrow 3 G$ & $41,58 a$ & $28,28 b$ & $30,12 b$ \\
\hline \multicolumn{5}{|c|}{ Tab. 1.4. Para aulas de solos no $(\Rightarrow)$ próprio nível dos opinantes } \\
\hline $1 G$ & $\Rightarrow 1 \mathrm{G}$ & $51,52 \mathrm{Aa}$ & $23,08 \mathrm{Ab}$ & $25,40 \mathrm{~B} \mathrm{~b}$ \\
\hline $2 \mathrm{G}$ & $\Rightarrow 2 \mathrm{G}$ & $41,57 \mathrm{~B} \mathrm{a}$ & $26,73 \mathrm{Ab}$ & $31,69 \mathrm{Ab}$ \\
\hline \multirow{2}{*}{\multicolumn{2}{|c|}{$\begin{array}{l}3 G \\
\text { Subtotal }\end{array}$}} & $41,58 \mathrm{~B} \mathrm{a}$ & $28,28 \mathrm{Ab}$ & $30,12 \mathrm{AB} b$ \\
\hline & & $44,27 \pm 16,35 a$ & $26,16 \pm 12,04 b$ & $29,55 \pm 10,23 b$ \\
\hline
\end{tabular}

Tab. 1.5. Média de aulas de solos em $(\Rightarrow) 1 \mathrm{G}, 2 \mathrm{G}$ e $3 \mathrm{G}$, na visão de todos os opinantes

$1,2,3 \mathrm{G}(92) \Rightarrow 1 \mathrm{G}$

$39,74 \pm 17,46 \mathrm{Aa} \quad 33,77 \pm 18,78 \mathrm{Ab}$

$26,46 \pm 11,38 \mathrm{Ac}$

$1,2 \mathrm{G}(67) \Rightarrow 2 \mathrm{G}$

$38,85 \pm 15,33 \mathrm{Aa}$

$30,91 \pm 14,84 \mathrm{Ab}$

$30,22 \pm 10,30 \mathrm{Ab}$

$3 G(25) \quad \Rightarrow 3 G$

$41,58 \pm 13,51 \mathrm{Aa}$

$28,28 \pm 13,07 \mathrm{Ab}$

$30,12 \pm 6,19 \mathrm{Ab}$

Tab. 1.6. Para aulas de solos em $(\Rightarrow) 1 G, 2 G$ e $3 G$, na visão de $3 G$

\begin{tabular}{|c|c|c|}
\hline$\Rightarrow 1 \mathrm{G}$ & $31,81 \mathrm{Ab}$ & $43,94 \mathrm{~A} a$ \\
\hline$\Rightarrow 2 \mathrm{G}$ & $34,29 \mathrm{~A} a b$ & $37,94 \mathrm{AB} a$ \\
\hline$\Rightarrow 3 \mathrm{G}$ & $41,58 \mathrm{Aa}$ & $28,28 \mathrm{~B} \mathrm{~b}$ \\
\hline
\end{tabular}

Tab. 1.7. Para aulas de solos em $(\Rightarrow) 1 \mathrm{G}$ e $2 \mathrm{G}$, na visão de $2 \mathrm{G}$

\begin{tabular}{|c|c|c|c|}
\hline$\Rightarrow 1 \mathrm{G}$ & $37,46 \mathrm{~A} a$ & $34,09 \mathrm{~A} \mathrm{ab}$ & $28,43 \mathrm{Ab}$ \\
\hline$\Rightarrow 2 G$ & $41,57 \mathrm{Aa}$ & $26,73 \mathrm{~B} \mathrm{~b}$ & $31,69 \mathrm{Ab}$ \\
\hline
\end{tabular}

Tab. 1.8. Para aulas de solos em $(\Rightarrow) 1 \mathrm{G}$, na visão destes

$1 \mathrm{G} \quad \Rightarrow 1 \mathrm{G} \quad 51,52 \mathrm{a} \quad 23,08 \mathrm{~b} \quad 25,40 \mathrm{~b}$

Tab. 1.9. Para aulas em nível de ensino imediatamente anterior ao do opinante

$\begin{array}{llccc}2 \mathrm{G} & \Rightarrow 1 \mathrm{G} & 37,46 \mathrm{Aa} & 34,09 \mathrm{Aab} & 28,43 \mathrm{Ab} \\ 3 \mathrm{G} & \Rightarrow 2 \mathrm{G} & 34,29 \mathrm{Aab} & 37,94 \mathrm{Aa} & 27,74 \mathrm{Ab} \\ \text { Subtotal } & & 36,28 \pm 13,26 \mathrm{a} & 35,53 \pm 16,60 \mathrm{a} & 28,17 \pm 10,89 \mathrm{~b}\end{array}$

Tab. 1.10. Fração de $\mathrm{CH}$ de aulas nos $(\Rightarrow)$ três ambientes segundo as opiniões de $1 \mathrm{G}, 2 \mathrm{G}$ e $3 \mathrm{G}$

$\begin{array}{llcr}1 \mathrm{G}(25 \times 1 \text { vez=25) } & 51,52 \pm 18,38 \mathrm{~A} \mathrm{a} & 23,08 \pm 10,74 \mathrm{~B} \mathrm{~b} & 25,40 \pm 10,69 \mathrm{Ab} \\ 2 \mathrm{G}(42 \times 2 \text { vezes }=84) & 39,51 \pm 14,32 \mathrm{~B} \mathrm{a} & 30,41 \pm 14,91 \mathrm{AB} \mathrm{b} & 30,06 \pm 11,90 \mathrm{Ab} \\ 3 \mathrm{G}(25 \times 3 \text { vezes }=75) & 35,89 \pm 15,62 \mathrm{~B} \mathrm{a} & 36,72 \pm 18,87 \mathrm{Aa} & 27,37 \pm 8,48 \mathrm{Ab}\end{array}$

Tab. 1.11. Média geral da fração de $\mathrm{CH}$ de aulas sobre solos nos $(\Rightarrow)$ três ambientes de ensino Todos (184) $\quad 39,67 \pm 16,16 \mathrm{a} \quad 31,98 \pm 16,76 \mathrm{~b} \quad 28,33 \pm 10,54 \mathrm{c}$

'Foi aplicado o Teste de Tukey $(\alpha<0,05)$, em que os valores sucedidos com letras minúsculas nas linhas entre quadrantes, maiúsculas nas colunas dentro do quadrante, iguais ou diferentes, indicam, respectivamente, não haver ou haver diferença significante, em ordem alfabética decrescente, entre as sugestões de carga horária (\%).

Fonte: Elaborado pelo autor. 
Rieder, A.

Revela-se que as aulas de campo sobre solos devem ter mais de $1 / 3$ da $\mathrm{CH}$ total (fração dominante), exceto na opinião de $3 \mathrm{G}$, que sugerem, para o Ensino Fundamental, uma fração pouco menor que $1 / 3$ da $\mathrm{CH}$ total.

Lopes (2008) também cita que alunos 3G de Ciências Biológicas pediam mais aulas de campo. Algumas tentativas de proporcionar mais aulas AC no ensino de solo têm sido experimentadas conforme Beck e Klamt (1988).

$\mathrm{O}$ aluno ainda muito jovem enquanto cursa $1 \mathrm{G}$ pode desejar mais contato com o fato real (objeto de estudo) em relação a sua opinião nas fases seguintes de escolarização e quando já está mais desenvolvido. Então, para obter o primeiro conhecimento escolar sobre solos, o aluno fica mais estimulado utilizando todos os seus sentidos de percepção para a novidade, e, entre os mais usados, estão: o tato (ex.: saber se o solo é pegajoso ou não, é duro ou não etc.), a visão (ex.: qual a cor do solo e como varia; como se situa o solo na paisagem e entre os demais elementos), o olfato (ex.: o solo tem cheiro? Etc.). Com isto, o aluno consegue fazer a sua representação escolar inicial de solo. Talvez por isto, na fase fundamental do ensino, os alunos prefeririam passar mais tempo em aulas de campo que ao estarem em níveis mais adiantados, quando já adquiriram um conhecimento prévio sobre solos. Então, sim, possuem mais questionamentos que requerem construção de respostas mais cognitivas. A oportunidade de interagir com a realidade associada com a abordagem teórica ajuda o raciocínio lógico no estudante, o que confere com estudos de Lage, Nogueira e Foresti (2006) referentes a alunos do Ensino Fundamental. As aulas de campo expõem os alunos ao fato real e fortificam o processo de aprendizagem, pronunciadamente, em alunos do Ensino Fundamental, o que está em concordância com abordagens de Seniciato e Cavassan (2004).

Talvez, a redução da fração de $\mathrm{CH}$ indicada para o nível $1 \mathrm{G}$, pelos opinantes de níveis mais adiantados, seja mais uma expressão de suas necessidades do momento em que respondiam, e não tanto daquilo que precisavam na ocasião de seu nível de ensino inicial.

\section{AC.2. Entre as opiniões para o seu próprio nível escolar-alvo}

A fração de $\mathrm{CH}$ expressivamente mais elevada para as aulas $\mathrm{AC}$ foi requerida, com destaque, pelos estudantes do Ensino Fundamental (1G: 51,5\% > 2G: 41,5\% $\cong 3 \mathrm{G}$ : $41,5 \%$ ) [Tab. 1.4]. O desejo dos opinantes é de que haja uma fração de $\mathrm{CH}$ predominante para as aulas AC, ou seja: entre quatro e cinco aulas AC para cada dez totais programadas.

\section{AC.3. Entre as médias opinadas aos níveis alvejados}

As frações de $\mathrm{CH}$ para as aulas $\mathrm{AC}$ sugeridas aos ensinos de $1 \mathrm{G}, 2 \mathrm{G}$ e $3 \mathrm{G}$ por seus opinantes [Tab. 1.5] foram similares $(39,6 \pm 16,1 \%)$ [Tab. 1.11]. Face a fração de $\mathrm{CH}$ menor indicada aos outros em relação à opinada ao próprio nível de ensino em questão, o valor médio obtido passou a ser menor também.

\section{AC.4. Entre as opiniões de uma mesma categoria escolar para os níveis alvejados}

Os opinantes $3 \mathrm{G}$ indicam que deve ser utilizada uma fração similar de carga horária $(35,8 \% \pm 15,6 \%)$ para aulas AC nos três níveis escolares $(1 \mathrm{G}, 2 \mathrm{G}$ e $3 \mathrm{G})$ no ensino de solos [Tab. 1.6]. Esta recomendação similar também é ratificada pelos opinantes $2 \mathrm{G}$ (39,5\% \pm $14,3 \%)$ para aulas AC ao seu nível $(2 \mathrm{G})$ e ao nível escolar anterior (1G). [Tab. 1.7]. Enquanto 
Fração ideal da carga horária ...

os opinantes $1 \mathrm{G}$ pediram que seja utilizada, para as aulas AC, em seu próprio nível de ensino, mais da metade da carga horária total $(51,5 \% \pm 18,3 \%)$ [Tab. 1.8]. É um desejo acentuado, que dificilmente se concretizará nas escolas brasileiras.

\section{AC.5. Entre as opiniões de categoria escolar imediatamente posterior ao nível alvejado}

Neste caso podiam opinar 2G e 3G. Indicaram uma fração similar de $\mathrm{CH}$ (36,2\% $\pm 13,1 \%$ ) para as aulas $\mathrm{AC}$ em $1 \mathrm{G}$ e $2 \mathrm{G}$, respectivamente [Tab. 1.9]. Ainda é uma fração dominante a favor do ambiente AC, mas já menor que a indicada para seus próprios níveis (Tab. 1.4).

AC.6. Entre as ponderações das três categorias de opinantes [Tab. 1.10]

A fração média de $\mathrm{CH}$ opinada por estudantes $1 \mathrm{G}$ para as aulas $\mathrm{AC}$ foi significativamente maior que as recomendadas por opinantes de escolaridade mais adiantada (1G: 51,5\% > 2G: $39,5 \% \cong 3 \mathrm{G}: 35,8 \%$ ), independente para que níveis de ensino fossem aplicados estas aulas. Em todos os casos, os de nível mais adiantado propunham frações menores para os níveis anteriores que os para seu próprio nível. $\mathrm{O}$ valor mais alto de $\mathrm{CH}$ requerida para seu próprio nível foi dos opinantes de $1 \mathrm{G}$. Isto explica a razão da média dos opinantes $2 \mathrm{G}$ e $3 \mathrm{G}$ ter proporcionado uma fração de $\mathrm{CH}$ significativamente menor que de $1 \mathrm{G}$.

\section{AS. Aulas em sala}

Estas são aulas clássicas, ministradas em uma sala para se dar aula. Em geral, neste ambiente, são utilizados, predominantemente: as informações que o professor detém e o espaço para acomodação dos participantes, recursos para escrever e/ou projetar. A sala é concebida para a concentração do estudante, ele prestar atenção e seguir as orientações do professor. Ali são desenvolvidas as disciplinas em que o aluno deve se portar disciplinadamente. A participação ativa dos alunos em aulas é de suma importância para o êxito educativo, pois exige provas através da reconstrução do que lhe é dito. Não sendo suficiente transmitir dados, informações e conhecimentos de outros. O êxito do professor dependerá muito dos estímulos dados aos alunos para buscarem o conhecimento através de seus próprios esforços. É aí que começa a necessidade de se libertar da clássica e restritiva sala de aula.

\section{AS.1. Entre as opiniões para um mesmo nível escolar-alvo}

Para as aulas AS, a fração de $\mathrm{CH}$ sugerida para o Ensino Fundamental divergiu entre os opinantes $(1 \mathrm{G}, 2 \mathrm{G}, 3 \mathrm{G})$. Os opinantes $1 \mathrm{G}$ indicaram uma $\mathrm{CH}$ menor que os de níveis mais adiantados (1G: 23,0\% < 2G: 34,09 <3G: 43,9\%) [Tab.1.1]. Enquanto para as aulas AS no Ensino Médio, os opinantes $2 \mathrm{G}$ indicaram $\mathrm{CH}$ menor $(26,7 \%)$ que a sugerida pelo nível mais adiantado (3G: 37,9\%) [Tab.1.2]. Já para as aulas AS em 3G, a CH pedida pelos estudantes deste nível foi de $28,2 \%$ [Tab.1.3]. O fato chama atenção e talvez isto sugira certa aversão a aulas AS, pois os opinantes ao seu nível de ensino pedem $\mathrm{CH}$ menor $(<1 / 3)$ que a indicada pelos mesmos para os níveis anteriores $(>1 / 3)$.

O aluno, enquanto cursa $1 \mathrm{G}$, está menos estimulado a relacionar-se abstratamente com um fato em relação a sua opinião para a mesma questão quando estiver nas fases seguintes de escolarização. Os prováveis mais frequentes contatos com a realidade (no caso, com 
Rieder, A.

solos) talvez possam explicar estas diferenças nos pleitos. Talvez por isto, na fase Fundamental do ensino, os alunos, ainda extremamente curiosos, neste nível (1G), prefeririam passar menos tempo em aulas de sala que o manifestado ao estarem já em níveis mais adiantados ( $2 \mathrm{G}$ e $3 G$ ), fase em que possuem mais questionamentos cognitivos, pertinentes à própria evolução da escolarização. O aumento da fração de $\mathrm{CH}$ indicada para o nível $1 \mathrm{G}$, na medida em que os opinantes avançam em nível de escolarização, pode ser uma expressão da demanda sentida no momento que estavam respondendo do que propriamente estiveram precisando no nível de ensino inicial.

A tendência dos opinantes é proporem aumento de $\mathrm{CH}$ para aulas AS aos níveis de ensino mais passados, na medida em que avançam na escolarização, e não, propriamente, ao nível em que estão. Para seu nível, propõem uma fração menor que $1 / 3$ da $\mathrm{CH}$ total, mas, para os níveis anteriores, recomendam mais que $1 / 3 \mathrm{da} \mathrm{CH}$ para aulas em sala.

\section{AS.2. Entre as opiniões para o seu próprio nível escolar-alvo}

A CH requerida para as aulas AS foi similar nos 3 níveis escolares (1G: $23,0 \% \cong$ 2G: $26,7 \% \cong 3 \mathrm{G}: 28,2 \%$ ) ou seja (média \pm desvio padrão: $26,6 \% \pm 12,0 \%$ ) [Tab.1.4]. Os opinantes pedem que as aulas AS, em seus respectivos níveis de ensino, devam ocupar uma fração minoritária da $\mathrm{CH}(<1 / 3$ do total). Contudo, esta tendência não se mantém quando opinam sobre a fração de $\mathrm{CH}$ para $\mathrm{AS}$ que deveria ser aplicada nos níveis anteriores à escolaridade dos opinantes.

\section{AS.3. Entre as médias opinadas aos níveis alvejados}

Também foi similar a $\mathrm{CH}$ sugerida para as aulas AS ao ensino de $1 \mathrm{G}, 2 \mathrm{G}$ e $3 \mathrm{G}$ por todos os seus opinantes [Tab. 1.5] (média \pm desvio padrão: 31,9\% $\pm 16,7 \%$ ) [Tab. 1.11]. O centro nesta faixa média, para aulas AS, está um pouco abaixo de $1 / 3$ da $\mathrm{CH}$ total.

\section{AS.4. Entre as opiniões de uma mesma categoria escolar para os níveis alvejados}

Os universitários $(3 \mathrm{G})$ opinaram que deveria ser utilizada, para as aulas AS, uma $\mathrm{CH}$ diferenciada para cada nível escolar (1G: 43,9\% $\geq 2 \mathrm{G}: 37,9 \% \geq 3 \mathrm{G}: 28,2 \%$ ) [Tab. 1.6], sendo a $\mathrm{CH}$, para $1 \mathrm{G}$ e $2 \mathrm{G}$, maior que $1 / 3$, enquanto menor para $3 \mathrm{G}$. Os opinantes do Ensino Médio (2G) também indicaram uma $\mathrm{CH}$ diferenciada, menos para o seu nível escolar (2G: 26,7\%) e mais para o nível anterior (1G: 34,0\%) [Tab. 1.7]. Esta tendência de os opinantes indicarem $\mathrm{CH}$ retrocrescente para aulas AS dos níveis anteriores se mostra recorrente, em oposição ao que ocorre com a CH para as aulas AC. Por sua vez, para os opinantes do Ensino Fundamental, o pedido é que seja aplicado, ao seu nível escolar, menos de $1 / 4 \mathrm{da} \mathrm{CH}$ total $(23,0 \% \pm 10,7 \%)$ [Tab. 1.8]. Isto pode significar que as aulas AS são menos atraentes.

\section{AS.5. Entre as opiniões de categoria escolar} imediatamente posterior ao nível alvejado

Neste caso, se enquadraram para opinar $2 \mathrm{G}$ e $3 \mathrm{G}$. Indicaram uma fração similar de $\mathrm{CH}(35,5 \% \pm 16,6 \%)$ para as aulas AS em 1G e 2G, respectivamente [Tab.1.9]. Esta fração é maior que a requerida para seu próprio nível escolar (Tab. 1.4). Isto expressa certa rejeição de aulas AS e, ao mesmo tempo, transferência do incômodo para outros. 
AS.6. Entre as ponderações das três categorias de opinantes [Tab. 1.10]

A média de fração de $\mathrm{CH}$ sugerida por universitários (3G) para as aulas AS foi significativamente maior que a opinada por $1 \mathrm{G}$, sendo que a indicada por $2 \mathrm{G}$ não se diferenciou dos colegas de outras escolaridades (3G: 36,7\% $\geq 2 \mathrm{G}: 30,4 \% \geq 1 \mathrm{G}: 23,0 \%$ ), independente de para que níveis escolares fossem aplicadas estas aulas [Tab. 1.10]. A fração de $\mathrm{CH}$ para as aulas AS mostra uma tendência crescente com o nível escolar. Isto ocorre, também, em face de os mais adiantados poderem opinar para níveis escolares anteriores e, recomendarem $\mathrm{CH}$ maior para esses, mas não propriamente para seu nível.

\section{AL - Aulas de laboratório}

Aulas de laboratório podem ajudar muito na melhoria da eficácia do ensino, mas as escolas precisam ter estrutura física, equipamentos, planejamento e servidores bem preparados, o que, em geral, não existe, conforme alerta o estudo de Caon (2005). Lopes (2008) revela manifestações de alunos de nível Superior (Ciências Biológicas) que requerem aulas práticas de laboratório por: complementarem a teoria; permitirem visualizar o foco do estudo; possibilitarem colocar teorias em prática, aprenderem com experimentos, tornando mais fácil, atrativo e rápido o processo de aprendizagem.

Boas condições para aulas de laboratório e a adequada aplicação destas no processo educativo certamente estimulam o surgimento de cientistas.

\section{AL.1. Entre as opiniões para um mesmo nível escolar-alvo}

Opinantes dos três níveis escolares indicaram que as aulas AL sobre solos, para qualquer nível de ensino, deveriam ter menos de $1 / 3 \mathrm{da} \mathrm{CH}$ total. Também foram similares as $\mathrm{CH}$ indicadas, pelos respectivos opinantes, para as aulas AL no Ensino Fundamental (1G: 25,4\% $\cong 2 \mathrm{G}: 28,4 \% \cong 3 \mathrm{G} 24,2 \%$ ) [Tab.1.1] e no Ensino Médio (2G: 31,6\% $\cong 3 \mathrm{G}: 27,7 \%$ ) [Tab. 1.2]. Para aulas AL em 3G, a fração da $\mathrm{CH}$ indicada por eles próprios foi de 30,1\% [Tab.1.3]. Aulas de laboratório permitem a verificação experimental de abordagens teóricas, facilitando a aprendizagem, conforme observa Lopes (2008). Para 1G, as indicações revelam que, aproximadamente, em cada quatro aulas no ensino de solos, uma deveria ser executada em laboratório.

\section{AL.2. Entre as opiniões para o seu próprio nível escolar-alvo}

A fração de $\mathrm{CH}$ mais elevada para as aulas $\mathrm{AL}$ foi requerida por estudantes $2 \mathrm{G}$, e a mais baixa por $1 \mathrm{G}$ (2G: $31,6 \% \geq 3 \mathrm{G}: 30,1 \% \geq 1 \mathrm{G}: 25,4 \%$ ) [Tab.1.4]. Em termos de CH, as aulas de laboratório não são as preferenciais para os três níveis e, menos ainda, para os estudantes $1 \mathrm{G}$.

\section{AL.3. Entre as médias opinadas para os níveis alvejados}

As $\mathrm{CH}$ para as aulas AL indicadas para os ensinos de $1 \mathrm{G}, 2 \mathrm{G}$ e $3 \mathrm{G}$, por seus opinantes [Tab. 1.5], foram similares $(28,3 \% \pm 10,5 \%)$ [Tab 1.11]. Esta média reforça que os estudantes dos três níveis consideram ser suficiente a utilização de menor $\mathrm{CH}$ para aulas AL em relação aos outros ambientes de aula, no ensino de solos. 
Rieder, A.

\section{AL.4. Entre as opiniões de uma mesma categoria escolar para os níveis alvejados}

As opiniões de $3 \mathrm{G}$ indicam que, para as aulas $\mathrm{AL}$, deveria ser utilizada uma fração diferenciada de carga horária para os três níveis escolares (1G: 24,2\% $\leq 2 \mathrm{G}: 27,7 \% \leq$ 3G: 30,1\%) [Tab. 1.6], enquanto $2 \mathrm{G}$ opinaram que deveria ser aplicada uma fração similar de CH $(30,4 \% \pm 14,9 \%)$ para o seu nível $(2 \mathrm{G})$ e o nível escolar anterior (1G) [Tab. 1.7]. Por sua vez, $1 \mathrm{G}$ opinaram que deveria ser utilizado, para as aulas $\mathrm{AL}$, em torno de $1 / 4 \mathrm{da} \mathrm{CH}$ total planejada $(25,4 \% \pm 10,6 \%)$ [Tab. 1.8$]$.

\section{AL.5. Entre as opiniões de categoria escolar} imediatamente posterior ao nível alvejado

Neste caso, se enquadraram para opinar $2 \mathrm{G}$ e $3 \mathrm{G}$. Indicaram uma fração similar de $\mathrm{CH}(28,1 \% \pm 10,8 \%)$ para as aulas AL em $1 \mathrm{G}$ e $2 \mathrm{G}$, respectivamente [Tab. 1.9].

\section{AL.6. Entre as ponderações das três categorias de opinantes}

As médias de fração de $\mathrm{CH}$ para as aulas AL sugeridas pelos estudantes $1 \mathrm{G}, 2 \mathrm{G}$ e $3 \mathrm{G}$ foram similares, situando-se entre $25-30 \%$ da $\mathrm{CH}$ total, independente de para que níveis escolares fossem aplicadas estas aulas [Tab. 1.10].

As opiniões, em geral, mostram que, no ensino de solos, as aulas em laboratório devem ser ministradas sem a utilização da mesma proporção de $\mathrm{CH}$ aplicada para as aulas dos outros ambientes de aula. Isto pode significar que a preferência pela aprendizagem sobre solos dos alunos não se dá através das aulas AL e/ou, então, a contribuição efetiva à aprendizagem das aulas AL requer menos tempo que os outros ambientes.

\section{Entre ambientes de aulas}

Há mudança de visão, em relação à distribuição da $\mathrm{CH}$ por ambiente de aula no ensino de solos, com a progressão escolar. Provavelmente, isto esteja relacionado às demandas e características próprias de cada fase de evolução no processo de descobertas e aprendizagem junto com o desenvolvimento da pessoa, desde criança, passando pela adolescência, juventude, e fase adulta. Entretanto, não há certeza sobre a razão da manifestação contraditória de indicarem uma fração de $\mathrm{CH}$ maior e menor, respectivamente, para as aulas AC e AL para seu nível atual de ensino em relação as suas recomendações para os níveis anteriores de ensino.

\subsection{G - No Ensino Fundamental (opinantes: 1G, 2G, 3G)}

Com exceção para as aulas AL, os opinantes dos três níveis escolares divergiram expressivamente nas indicações da distribuição da $\mathrm{CH}$ para aulas AC e AS sobre solos no ensino lG: os opinantes do próprio $1 \mathrm{G}$ requerem muito mais aulas AC que AS, enquanto os opinantes de níveis mais adiantados, progressivamente, reduzem a $\mathrm{CH}$ de aulas AC indicadas a esses e aumentam as de AS.

\section{A. Conforme o nível escolar dos opinantes}

Os estudantes $1 \mathrm{G}$ recomendam, para seu próprio nível, frações de $\mathrm{CH}$ distintas aos ambientes de aula: mais aulas AC e proporções menores, mas similares, de aulas AL e AS 
Fração ideal da carga horária ...

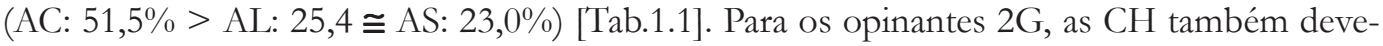
riam ser distintas no ensino de solos em $1 \mathrm{G}$, com mais aulas de campo e menos de laboratório (AC: $37,4 \%>$ AS: 34,0\% > AL: 28,4\%) [Tab.1.1]. Esta $\mathrm{CH}$, embora ainda predominante, é menos expressiva que a requerida pelos opinantes do próprio nível Fundamental, cujo contraste foi compensado às aulas $\mathrm{AS}$, que aumentaram. Enquanto os opinantes $3 \mathrm{G}$ sugeriram, para $1 \mathrm{G}$, mais aulas AS e proporções estatisticamente similares, mas, menores, de aulas de

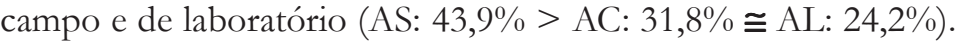

\section{B. Independente do nível escolar dos opinantes}

No ensino $1 \mathrm{G}$ sobre solos, as médias de fração de $\mathrm{CH}$ sugeridas por todos $(1 \mathrm{G}$, $2 \mathrm{G}$ e $3 \mathrm{G}$ juntos) foram distintas nos três ambientes de aula, sendo requerida mais de aula de campo e menos de laboratório (AC: 39,7\% > AS: 33,7\% > AL: 26,4\%) [Tab.1.5]. As médias de $\mathrm{CH}$ para as aulas de AC e AS derivam de expressiva variabilidade de opinião, enquanto as médias de aulas AL resultam de opiniões mais homogêneas.

\subsection{G - No Ensino Médio (opinantes: 2G, 3G)}

\section{A. Conforme o nível escolar dos opinantes}

Os do próprio nível $(2 \mathrm{G})$ requerem proporções distintas de $\mathrm{CH}$, maior para aulas

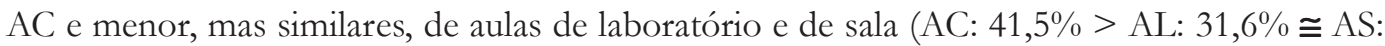
$26,7 \%$ ) [Tab.1.2], enquanto as recomendadas pelo nível Superior ( $3 G)$ invertem esta ordem, sugerindo mais aulas AS e menos AL (AS: 37,9\% $\geq$ AC: 34,2\% $\geq$ AL: 27,7\%) [Tab.1.2]. Estas discordâncias, provavelmente, possam ser explicadas por efeitos da própria escolarização, das experiências e da aprendizagem proporcionadas com o avanço etário das pessoas.

\section{B. Independente do nível escolar dos opinantes}

Em 2G, a média de fração de $\mathrm{CH}$ sugerida pelos opinantes ( $2 \mathrm{G}$ e $3 \mathrm{G}$ ) para aulas AC foi maior que para as aulas AS e AL (AC: $38,8 \%>$ AS: $30,9 \% \cong$ AL: 30,2\%) [Tab.1.5].

\subsection{G - No Ensino Superior (opinantes: apenas 3G)}

Os estudantes $3 \mathrm{G}$ indicaram que as frações de $\mathrm{CH}$ destinadas aos três ambientes de aula deveriam ser distintas no ensino de solos na Educação Superior: mais aulas AC e propor-

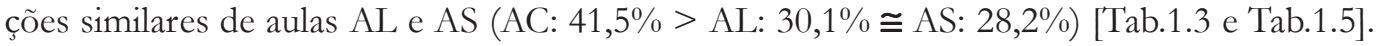

\section{Médias $(\mu)$}

\section{3 - $\mu 123$ G123 - Média das opiniões para o seu próprio nível}

As aulas AC receberam uma fração média expressivamente maior de $\mathrm{CH}(44,2 \% \pm$ $16,3 \%$ ) em relação à fração similar recomendada aos outros ambientes (AS: $26,1 \% \pm 12,0 \% \cong$ AL:29,5\% \pm 10,2\%) [Tab.1.4]. 
Rieder, A.

3 - $\mu 23$ G12 - Média das indicações

para o nível imediatamente anterior ao do opinante

As opiniões provindas dos níveis $2 \mathrm{G}$ e $3 \mathrm{G}$, respectivamente, para $1 \mathrm{G}$ e $2 \mathrm{G}$, derivaram uma fração média de $\mathrm{CH}$ menor para aulas $\mathrm{AL}(27,3 \% \pm 8,4 \%)$ e maior, mas similar, para as aulas dos outros dois ambientes (AS: $36,7 \% \pm 18,8 \% \cong$ AC: $35,8 \% \pm 15,6 \%$ ) [Tab.1.9]. Ao opinarem para seu próprio nível, aumentaram a fração destinada para aulas AC e reduziram, expressivamente, a $\mathrm{CH}$ para aulas AS [Tab.1.4].

\section{3 - H3G - Média das opiniões de 3G}

As opiniões provindas do nível $3 \mathrm{G}$ propiciaram uma fração média de $\mathrm{CH}$ menor para aulas $\operatorname{AL}(28,1 \% \pm 10,8 \%)$ e maior, mas similar, para as aulas dos outros dois ambientes (AS:35,5\% $116,6 \% \cong \mathrm{AC}: 36,2 \% \pm 13,2 \%$ ) [Tab.1.6 e 1.10$]$.

3 - $\mu 2 \mathrm{G}$ - Média das opiniões de $2 \mathrm{G}$

As opiniões emanadas do nível $2 \mathrm{G}$ resultaram em uma fração média de $\mathrm{CH}$ similarmente menor para aulas AL $(30,0 \% \pm 11,9 \%)$ e AS $(30,4 \% \pm 14,9 \%)$, enquanto maior para as aulas AC (39,5\% \pm 14,3\%) [Tab.1.7 e 1.10].

3 - $\mu 1 \mathrm{G}$ - Média das opiniões de $1 \mathrm{G}$

As frações médias de $\mathrm{CH}$ obtidas das opiniões de $1 \mathrm{G}$ confirmam, de modo mais acentuado (AL: $25,4 \cong \mathrm{AS}: 23,0 \%<\mathrm{AC}: 51,5 \%$ ), a tendência das opiniões expressas por $2 \mathrm{G}$ (II. $\mu 2 \mathrm{G})$ [Tab.1.1, 1.8 e 1.10].

$3-\mu$ Geral

As médias das frações de $\mathrm{CH}$ sugeridas, no geral, nos três ambientes de aula no ensino de solos, foram distintas, sendo requeridas mais aulas de campo, menos de laboratório e uma fração mediana para aulas em sala (AC: 39,6\% > AS: 31,9\% > AL: 28,3\%).

Em qualquer caso analisado, no ensino de solos, a $\mathrm{CH}$ não deve ser igualitária entre ambientes de aula, e sim deve privilegiar as aulas de campo.

\section{Conclusões}

O nível de escolaridade dos estudantes afeta as opiniões sobre a carga horária ideal $(\mathrm{CH})$ preconizada para o exercício das estratégias didático-pedagógicas de "campo", "sala de aula" e de "laboratório" no ensino de temas relacionados a solos.

Em qualquer nível escolar, os estudantes manifestam que as aulas sobre solos devem ser desenvolvidas não apenas num único ambiente, mas em sala (AS), campo (AC) e laboratório (AL). Requerem que as frações de $\mathrm{CH}$ para as aulas nos três ambientes de ensino não devam ser iguais. Apontam que as aulas de laboratório devem ter menos $\mathrm{CH}$ (na faixa de $1 / 4$ a $1 / 3$ do total), enquanto as aulas de campo mais (na faixa de $1 / 3$ a $1 / 2$ do total), exceto para o Ensino Fundamental (1G), no pensamento dos opinantes do Ensino Superior (3G).

As menores proporções de $\mathrm{CH}$ para as aulas de campo, em sala e laboratório foram sugeridas, respectivamente, por opinantes dos ensinos 3G (Superior), 1G (Fundamental) e 3G 
Fração ideal da carga horária ...

(Superior), concernentemente, destinadas todas para o mesmo nível escolar: $1 \mathrm{G}$; enquanto as maiores foram indicadas, respectivamente, por $1 \mathrm{G}, 3 \mathrm{G}$ e $2 \mathrm{G}$, concernentemente, designadas para os níveis escolares $1 G, 1 G$ e $2 G$.

As frações de $\mathrm{CH}$ para aulas em sala, sugeridas para níveis anteriores, são maiores que as opinadas para seu próprio nível escolar, enquanto ocorre o inverso com a $\mathrm{CH}$ para as aulas de campo. Estas discrepâncias podem estar indicando mais rejeição às aulas em sala e maior atratividade das aulas de campo para o próprio nível escolar dos opinantes, e, ao mesmo tempo, transferem mais carga rejeitada para os níveis anteriores, com suas opiniões.

Levanta-se a hipótese de que as aulas em sala podem significar, para os estudantes, um mal necessário; enquanto o inverso ocorre com as aulas de campo que, por sua vez, se expressa como algo bem-vindo.

Os estudantes dos três níveis escolares preconizaram, para seu nível, uma fração de $\mathrm{CH}$ maior para aulas de campo que a fração de tempo destinada para aulas em sala e laboratório, no ensino de solos.

\section{Referências}

ALVES, M. B. N.; ELÓY, C. A. S.; PEREIRA, M. A. S. Desenvolvendo os sentidos para a educação ambiental: atividades práticas no ensino fundamental. In: ENCUENTRO DE GEÓGRAFOS DE AMÉRICA LATINA, 12., 2009, Montevideo. Anais... Montevideo, Universidad de la República, 2009. Disponível em: <http:// observatoriogeograficoamericalatina.org.mx/egal12/Procesosambientales/Usoderecursos/ 04.pdf >. Acesso em: 07 abr. 2011.

ARAÚJO, C. H.; CONDE, F. N.; LUZIO, N. Índice de qualidade da educação fundamental (IQE): proposta para discussão. Revista Brasileira de Estudos

Pedagógicos, Brasília, v. 85, n. 209/210/211, p. 126-136, jan./dez. 2004. Disponível em: <http://www.rbep.inep.gov.br /index.php/RBEP/article/viewFile/94/96> Acesso em: 24 mar. 2011.

BECK, F. L.; KLAMT, E. Organização do conteúdo do ensino de solos e sua relação com a sociedade. In: MONIZ, A. C. (Ed.). A responsabilidade social da ciência do solo. Campinas: Sociedade Brasileira de Ciência do Solo, 1988. p. 169-181.

BRANDÃO, C. R. O que é educação. 3. ed. São Paulo: Brasiliense, 1981.

BRASIL. Presidência da República. Lei No 5.692, de 11 de agosto de 1971. Fixa diretrizes e bases para o ensino de $1^{\circ}$ e 2 graus, e dá outras providências. Disponível em: <http:// www.planalto.gov.br/ccivil_03/leis/L9394.htm\#art92>. Brasília, 1971. Acesso em: 05 fev. 2014.

Presidência da República. Lei $\mathbf{N}^{\circ}$ 9.394, de 20 de dezembro de 1996. Estabelece as diretrizes e bases da educação nacional. Brasília, 1996. Disponível em: <http:/ / www.planalto.gov.br/ccivil_03/leis/L9394.htm\#art92>. Acesso em: 05 fev. 2014. 
Rieder, A.

CAON, C. M. Concepções de professores sobre o ensino e a aprendizagem de ciências e de biologia. 2005. 94 f. Dissertação (Mestrado em Educação em Ciências e Matemática) - Pontifícia Universidade Católica do Rio Grande do Sul, Porto Alegre, 2005. Disponível em: <http://www.educadores.diaadia.pr.gov.br/arquivos/File/2010/ artigos_teses/2010/Biologia/dissertacoes/concepcao_professores.pdf $>$. Acesso em: 06 jul. 2013.

CAPUCHO, J. d'O. A natureza na aprendizagem científica: o percurso pedestre como instrumento de um ambiente educativo: o Parque Natural de Sintra-Cascais. 2009. Dissertação (Mestrado em Ciências) - Faculdade de Ciências, Universidade de Lisboa, Lisboa, 2009. Disponível em: < http://repositorio.ul.pt/handle/10451/3582>. Acesso em: 16 set. 2011.

CATANOZI, G. Uma proposta de material didático sobre solos para o ensino médio e técnico. 2004. 140 f. Dissertação (Mestrado em Tecnologia) - Centro Estadual de Educação Tecnológica Paula Souza, São Paulo, 2004. Disponível em: <http:// www.centropaulasouza.sp.gov.br/posgraduacao/Trabalhos/Dissertacoes/ DM_Tecn_Gerson_Catanozi.pdf>. Acesso em: 01 jan.2011.

CECCON, C.; OLIVEIRA, M. D. de; OLIVEIRA, R. D. de. A vida na escola e a escola da vida. 4. ed. Petrópolis: Vozes: IDAC, 1982.

COMPIANI, M.; DAL RÉ CARNEIRO, C. Os papéis didáticos das excursões geológicas. Enseñanza de las Ciencias de la Tierra, Girona, v. 1, n. 2, p. 90-97, 1993.

COSTA, L. M. da; DIAS, L. E. O ensino de solos na Universidade Federal de Viçosa. In: MONIZ, A. C. (Ed.). A responsabilidade social da ciência do solo. Campinas: Sociedade Brasileira de Ciência do Solo, 1988. p. 183-186.

DINIZ, A. A.; BATISTA, R. B.; SANTOS, R. F. dos. Popularização da taxonomia de solo: vocabulário mínimo e aspectos sócio-econômicos no contexto do ensino fundamental - São Miguel, Esperança (PB). Revista Brasileira de Ciência do Solo, Viçosa, v. 29, n. 2, p. 309-316, 2005.

DORIN, L. Livro-texto de psicologia da educação. São Paulo: Brasil, 1978.

FALCONI, S. A produção de material didático para o ensino de solos. 2004. 115 f. Dissertação (Mestrado em Geografia) - Universidade Estadual Paulista, Rio Claro, 2004.

FERREIRA, M. G. V. X. Ensino de solos: uma visão global. In: MONIZ, A. C. (Ed.). Responsabilidade social da ciência do solo. Campinas: Sociedade Brasileira de Ciência do Solo, 1988. p. 187-191.

FONSECA G. da; CALDEIRA, A. M. de A. Uma reflexão sobre ensino aprendizagem de ecologia em aulas práticas e a construção de sociedades sustentáveis. Revista Brasileira de Ensino de Ciência e Tecnologia, Ponta Grossa, v. 1, n. 3, p. 70-92, set-dez 2008. Disponível em: < http://revistas.utfpr.edu.br/pg/index.php/rbect/article/view/240>. Acesso em: 17 dez. 2011. 
Fração ideal da carga horária ...

FRASSON, V. da R.; WERLANG, M. K. Ensino de solos na perspectiva da educação ambiental: contribuições da ciência geográfica. Geografia: ensino \& pesquisa, Santa Maria, v. 14, n. 1, p. 94- 99, 2010.

GOLGHER, A. B. Diálogos com o ensino médio 2: o ensino médio no Brasil visto a partir do modelo profluxo e outros indicadores demográficos. Belo Horizonte: UFMG: Cedeplar, 2010.

INEP. Censo da educação superior: sinopse 1995 a 2009. Disponível em: <http:// inep.gov.br/superior/censosuperior/sinopse/1995/default.htm>. Acesso em: 23 mar. 2011.

LAGE, F.; NOGUEIRA, M. G.; FORESTI, M. C. P. A importância do tema água doce no ensino fundamental: uma proposta de aulas teórico-práticas. In: PINHO, S. Z. de;

SAGLIETTI, J. R. C. (Org.). Núcleos de ensino. São Paulo: UNESP, 2006. v. 1, p. 49-68. Disponível em: <http://www.unesp.br/prograd/PDFNE2004/artigos/eixo2/

aimportanciadotemaguadoce.pdf >. Acesso em: 02 set. 2011.

LIMA, M. R. de (Ed.). Pesquisa e popularização da educação em solos. In: SIMPÓSIO BRASILEIRO DE EDUCAÇÃO EM SOLOS, 5., 2010, Curitiba. Resumos... Curitiba: Universidade Federal do Paraná; Sociedade Brasileira de Ciência do Solo, 2010. Disponível em: < http://www.sbes.ufpr.br/resumos_expandidos_VSBES.pdf >. Acesso em: 07 abr. 2011.

LIMA, M. R. de. O solo no ensino de ciências no nível fundamental. Ciência \& Educação, Bauru, v. 11, n. 3, p. 383-395, 2005. Disponível em: <http://www.scielo.br/ scielo.php?script $=$ sci_arttext\&pid=S1516-73132005000300004\&lng=en\&nrm=iso $>$. Acesso em: 07 abr. 2011.

LOPES, M. C. P. Proposição de metodologias inovadoras com a utilização de recursos de aprendizagem na area de ciências biológicas. 2008. 178 f. Dissertação (Mestrado em Educação) - Pontifícia Universidade Católica do Paraná, Curitiba, 2008.

MARCOS, Z. Z. Ensino agronômico: intuitivo ou metódico? In: MONIZ, A. C. (Ed.). A responsabilidade social da ciência do solo. Campinas: Sociedade Brasileira de Ciência do Solo, 1988. p. 203.

MELLO, G. N. de. Fatores intra-escolares como mecanismo de seletividade no ensino de $1^{\circ}$ grau. Educação \& Sociedade, Campinas, v. 1, n.2, p.70-78. 1979.

MIDÃO, C. M. de V.; RUIZ-MORENO, L. O ensino da semiologia nas escolas médicas do estado do Rio de Janeiro. Revista Brasileira de Educação Médica, Rio de Janeiro, v. 34, n. 3, p. 397-405, 2010. Disponível em: <http://www.scielo.br/ scielo.php?script $=$ sci_arttext\&pid $=\mathrm{S} 0100-55022010000300009 \& \operatorname{lng}=$ en\&nrm $=$ iso $>$. Acesso em: 24 mar. 2011.

MUGGLER, C. C; PINTO SOBRINHO, F. A; MACHADO, V. A. Educação em solos: princípios, teoria e métodos. Revista Brasileira de Ciência do Solo, Campinas, v. 30, n. 4, p.733-740, 2006. 
Rieder, A.

PENTEADO, W. M. A.(Org.). Psicologia e ensino. São Paulo: Papelivros, 1980.

PESSOA, R. B. Um olhar sobre a trajetória da geografia escolar no Brasil e a visão dos alunos de ensino médio sobre a geografia atual. 2007. 130 f. Dissertação (Mestrado em Geografia) - Universidade Federal de Paraíba, João Pessoa, 2007. Disponível em: < http://www.geociencias.ufpb.br/posgrad/dissertacoes/ rodrigo_pessoa.pdf $>$. Acesso em: 15 set. 2011.

PRIEVE, P. E.; LISOVSKI, L. A. Uso do Parque Municipal Miguel Pereira pelos professores de ciências e biologia de Roncador - PR. Cadernos da Pedagogia, São Carlos, v. 4, n. 7, p. 111-124, 2010. Disponível em: <http:// www.cadernosdapedagogia.ufscar.br/index.php/cp/article/viewFile/181/107>. Acesso em: 15 set. 2011.

REICHARDT, K. Por que estudar o solo? In: MONIZ, A. C. (Ed.). A responsabilidade social da ciência do solo. Campinas: Sociedade Brasileira de Ciência do Solo, 1988. p. $75-78$.

REZENDE D. M.; PIRES L. M. A visão dos alunos do ensino médio sobre o ensino de geografia: um estudo de caso do Instituto Federal Goiano - campus Morrinhos. In:

ENCONTRO NACIONAL DE PRÁTICA DE ENSINO EM GEOGRAFIA, 10., 2009, Porto Alegre. Anais... Porto Alegre: AGB, 2009. Disponível em: <http://www.agb.org.br/ XENPEG/artigos/GT/GT4/tc4\%20(21).pdf>. Acesso em: 03 abr. 2011.

RIGHETTTO, M. Educação de jovens e adultos: uma discussão sobre a formação docente. 2007. 148 f. Dissertação (Mestrado em Educação) - Centro de Ciências da Educação, Universidade Regional de Blumenau, Blumenau, 2007. Disponível em: <http:// proxy.furb.br/tede/tde_busca/arquivo.php?codArquivo=489>. Acesso em: 13 set. 2011.

RUELLAN, A. Pedologia e desenvolvimento: a ciência do solo ao serviço do desenvolvimento. In: MONIZ, A. C. (Ed.). A responsabilidade social da ciência do solo. Campinas: Sociedade Brasileira de Ciência do Solo, 1988. p. 69-74.

SENICIATO, T.; CAVASSAN, O. Afetividade, motivação e construção de conhecimento científico nas aulas desenvolvidas em ambientes naturais. Ciências \& Cognição, Rio de Janeiro, v. 13, n. 3, p.120-136. 2008.

. Aulas de campo em ambientes naturais e aprendizagem em Ciências - um estudo com alunos do ensino fundamental. Ciência \& Educação, Bauru, v. 10, n. 1, p. 133-147, 2004. Disponível em: <www.scielo.br/pdf/ciedu/v10n1/10.pdf>. Acesso em: 01 set. 2011.

SENICIATO, T.; SILVA, P. G. P. da; CAVASSAN, O. Construindo valores estéticos nas aulas de ciências desenvolvidas em ambientes naturais. Ensaio, Belo Horizonte, v. 8, n. 2 p. 97-109, 2006. Disponível em: <http://www.portal.fae.ufmg.br/seer/index.php/ensaio/ article/viewFile/110/205> Acesso em: 16 set.11

SILVA JUNIOR, C.; SASSON, S.; SANCHES, P. S. B. Ciências: entendendo a natureza - o mundo em que vivemos. 6. ed. São Paulo: Saraiva, 1995. 
Fração ideal da carga horária ...

SILVA, P. M.; FONTINHA S. R. Ciências, $1^{\text {o }}$ grau. São Paulo: Companhia Editora Nacional, [19-]. v. 1.

TAVARES, A. D. O ensino da física. Rio de Janeiro: NEPEC, 1960.

TRANI, P. E. O ensino e a pesquisa em solos vistos pelo extensionista. In: MONIZ, A. C. (Ed.). A responsabilidade social da ciência do solo. Campinas: Sociedade Brasileira de Ciência do Solo, 1988. p. 197-201. 
Rieder, A.

Apêndice A. Formulário: estudos sobre o ensino de solos no 1ํㅜ 2ํㅡ e 3ํㅡㅁaus, Mato Grosso, 1995.

Questão I. Identificação: (Nome codificado - ordem numérica)
1. Idade:
..anos
2. Sexo:.......
3. Curso:
4. Semestre/ano:
4. Instituição:
6. Data:............./1995.

Questão II: Como deve ser a distribuição ideal de carga horária de aulas no ensino de solos?

- Para estudantes do $1^{\circ}$ grau:

1. Para melhorar a aprendizagem e o interesse do aluno no estudo de solos (ensino de $1^{\circ} \mathrm{grau}$ ), em 100 horasaula, quantas horas devem ser usadas nos seguintes ambientes de aula?

Em sala de aula:....hs $\quad$ Em laboratório:...hs $\quad$ Ao vivo, no campo:...hs $\quad$ Total: $100 \mathrm{hs}$

- Para estudantes do $2^{\circ}$ grau:

2. Para melhorar a aprendizagem e o interesse do aluno no estudo de solos (ensino de $1^{\circ}$ e $2^{\circ}$ graus), em 100 horas-aula, quantas devem ser usadas nos seguintes ambientes de aula?

\begin{tabular}{|c|c|c|}
\hline \multirow[b]{2}{*}{ Ambientes de aula } & \multicolumn{2}{|c|}{ Por nível de ensino, indicar a carga horária em \% } \\
\hline & 19 Grau & 2 Grau \\
\hline Sala de aula & & \\
\hline Laboratório & & \\
\hline Ao vivo, no campo & & \\
\hline Total & $100 \%$ & $100 \%$ \\
\hline
\end{tabular}

- Para estudantes do $3^{\circ}$ grau:

3. Para melhorar a aprendizagem e o interesse do aluno no estudo de solos (ensino de $1^{\circ}, 2^{\circ}$ e $3^{\circ}$ graus), em 100 horas-aula, quantas devem ser usadas nos seguintes ambientes de aula?

\begin{tabular}{|l|c|c|c|}
\hline \multirow{2}{*}{ Ambientes de aula } & \multicolumn{2}{|c|}{ Por nível de ensino, indicar a carga horária em \% } \\
\cline { 2 - 4 } & $\mathbf{1}$ \% Grau & $\mathbf{2}$ - Grau & \\
\hline Sala de aula & & & \\
\hline Laboratório & & & \\
\hline Ao vivo, no campo & & & $100 \%$ \\
\hline Total & $100 \%$ & $100 \%$ & \\
\hline
\end{tabular}

Artigo recebido em 12/07/13. Aceito em 16/12/13.

226

Ciênc. Educ., Bauru, v. 20, n. 1, p. 207-226, 2014 